\title{
Targeting the Sortase A Transpeptidase to Tackle Gram-positive Pathogens
}

\section{Domenico Schillaci *}

Department of Biological, Chemical, Pharmaceutical Science and Technology, Università degli Studi di Palermo, Via Archirafi, 32-90123 Palermo, Italy

Antibiotic resistance is a very important challenge and in 2011 the World Health Organization (WHO) considered it as one of the most important global health problems [1].

The emergence of drug resistance of Gram-positive pathogens such as Staphylococcus aureus is currently a health problem of global significance and the overuse of antibiotics, both in human and animal populations, played an important part in the beginning of drugresistant strains.

S. aureus is a very versatile pathogen that can cause a wide variety of infectious diseases ranging from superficial skin infections to lifethreatening endocarditis and sepsis [2]. Its versatility as a pathogen is due to the wide arsenal of weapons (virulence factors) that it possesses [3].

The virulence factors include the cell-wall associated proteins called MSCRAMMs (microbial surface components recognizing adhesive matrix molecules) that can promote the adhesion to host proteins, for example, the fibronectin that is very common in host tissues. The cellwall associated proteins are necessary for host colonization, invasion, immune evasion and biofilm formation [4]. The biofilms, multistratified bacterial communities that grow on a biological or artificial surface, are responsible for chronic infectious diseases and for device or biomaterial associated infections, and are more resistant to host immune defence system and to conventional antibiotics [5].

The cell-wall associated proteins are covalently linked to the peptidoglycan through the enzymatic activity of so-called sortases. These sortases are membrane-bound transpeptidases that cleave the peptide bond between the threonine (Thr) and the glycine residues of a LPxTG motif, and catalyze the formation of an amide bond between the carboxylic group of Thr residue and the free amino end of a pentaglycine cross bridge in peptidoglycan precursors [6].

The Sortase A (SrtA) is the most well studied member of the sortase family. S. aureus strains lacking the $S r t A$ gene do not present the LPxTG proteins at the cell surface. As a consequence, SrtA mutant strains are less virulent than wild strains and they are defective in the establishment of infections [7]. There are around 20 staphylococcal proteins that carry a C-terminal LPxTG motif, these include protein A (Spa), two fibronectin binding proteins (FnbpA and FnbpB) and two clumping factors (ClfA and ClfB). For some of these proteins a direct role in biofilm formation has been reported [8].

There is an urgent need of anti-virulence agents effective in the prevention or eradication of biofilms that are intrinsically resistant to conventional antibiotics. If we consider that the first step of staphylococcal pathogenesis and of biofilm formation is the bacterial adhesion, promoted by the surface exposed proteins at the cell wall, we believe that new anti-virulence agents could be developed by using as a target the enzyme responsible of linking surface exposed proteins rather than any single surface protein involved in the mechanism of virulence [9]. Therefore, the $S r t A$ is a good target to design novel antiinfective drugs and $S r t A$ inhibitors could act as anti-adhesion agents useful to prevent a virulence mechanism as biofilm formation.

Many inhibitors of StrA, afforded via high-throughput screening of libraries of compounds (synthetic or natural), have been identified, and most of them are useful for explaining the action model of the enzyme. Some of them are, instead, potential leading compounds [10]. The efficacy of the most interesting SrtA inhibitors needs to be evaluated by in vivo models of infection (sepsis, abscess, septic arthritis and endocarditis), but a simple methodology in vitro, such as inhibition of biofilm formation, could represent a first assessment of anti-virulence properties of novel inhibitors of SrtA.

There are some other important aspects concerning SrtA on which attention should be focused: other Gram positive pathogens like enterococci or streptococci possess the enzyme $\operatorname{SrtA}$; $\operatorname{SrtA}$ is not indispensable for microbial growth and survival so the $S r t A$ inhibitors have a limited antibacterial activity that could minimize the development of resistance; sortases are membrane enzymes that could be more easily targeted by inhibitors respect to intracellular targets [11].

The goal of the research in this field is to obtain novel SrtA inhibitors that could interfere with Gram positive virulence mechanisms including adhesion to host tissues, evasion of host defence, as phagocytosis or opsonisation, and the biofilm formation.

The possibility of using novel agents targeting virulence mechanisms and biofilm formation (anti-virulence agents) in combination with current antibiotics is a potential new therapeutic strategy in the treatment of bacterial chronic infections and can contribute to overcoming antibiotic resistance, which is considered one of today's most important global challenges.

\section{Acknowledgment}

The Author would like to thank Valeria R. Casale of On Point Translation for English revision of the manuscript.

\section{References}

1. http://www.who.int/world-health-day/2011/WHD201_FS_EN.pdf

*Corresponding author: Domenico Schillaci, Department of Biological, Chemical, Pharmaceutical Science and Technology, Università degli Studi di Palermo, Via Archirafi, 32-90123 Palermo, Italy, E-mail: domenico.schillaci@unipa.it

Received February 22, 2013; Accepted February 25, 2013; Published February 28,2013

Citation: Schillaci D (2013) Targeting the Sortase A Transpeptidase to Tackle Gram-positive Pathogens. J Microb Biochem Technol 5: e114. doi:10.4172/19485948.1000e114

Copyright: @ 2013 Schillaci D. This is an open-access article distributed under the terms of the Creative Commons Attribution License, which permits unrestricted use, distribution, and reproduction in any medium, provided the original author and source are credited 
Citation: Schillaci D (2013) Targeting the Sortase A Transpeptidase to Tackle Gram-positive Pathogens. J Microb Biochem Technol 5: e114. doi:10.4172/1948-5948.1000e114

Page 2 of 2

2. Lowy FD (1998) Staphylococcus aureus infections. N Engl J Med 339: 520-532

3. Tang YW, Stratton CW (2010) Staphylococcus aureus: An old pathogen with new weapons. Clin Lab Med 30: 179-208.

4. Scott JR, Barnett TC (2006) Surface proteins of gram-positive bacteria and how they get there. Annu Rev Microbiol 60: 397-423.

5. Høiby N, Bjarnsholt T, Givskov M, Molin S, Ciofu O (2010) Antibiotic resistance of bacterial biofilms. Int J Antimicrob Agents 35: 322-332.

6. Fischetti VA, Pancholi V, Schneewind O (1990) Conservation of a hexapeptide sequence in the anchor region of surface proteins from gram-positive cocci. Mol Microbiol 4: 1603-1605

7. Mazmanian SK, Liu G, Jensen ER, Lenoy E, Schneewind O (2000) Staphylococcus aureus sortase mutants defective in the display of surface proteins and in the pathogenesis of animal infections. Proc Natl Acad Sci USA 97: 5510-5515.
8. Tsompanidou E, Denham EL, Sibbald MJ, Yang XM, Seinen J, et al. (2012) The sortase A substrates FnbpA, FnbpB, ClfA and ClfB antagonize colony spreading of Staphylococcus aureus. PLoS One 7: e44646.

9. Chen L, Wen YM (2011) The role of bacterial biofilm in persistent infections and control strategies. Int J Oral Sci 3: 66-73.

10. Maresso AW, Wu R, Kern JW, Zhang R, Janik D, et al. (2007) Activation of inhibitors by sortase triggers irreversible modification of the active site. J Biol Chem 282: 23129-23139.

11. Fitzgerald-Hughes D, Devocelle M, Humphreys H (2012) Beyond conventional antibiotics for the future treatment of methicillin-resistant Staphylococcus aureus infections: two novel alternatives. FEMS Immunol Med Microbiol 65 399-412. 